\title{
The future African workplace: The use of collaborative robots in manufacturing
}

\begin{tabular}{|c|c|}
\hline \multicolumn{2}{|c|}{$\begin{array}{l}\text { Authors: } \\
\text { Andre P. Calitz } \\
\text { Paul Poisat }{ }^{2} \\
\text { Margaret Cullen }\end{array}$} \\
\hline \multicolumn{2}{|c|}{$\begin{array}{l}\text { Affiliations: } \\
{ }^{1} \text { Department of Computing } \\
\text { Sciences, Nelson Mandela } \\
\text { Metropolitan University, } \\
\text { South Africa }\end{array}$} \\
\hline \multicolumn{2}{|c|}{$\begin{array}{l}{ }^{2} \text { Nelson Mandela } \\
\text { Metropolitan University } \\
\text { Business School, Port } \\
\text { Elizabeth, South Africa }\end{array}$} \\
\hline \multicolumn{2}{|c|}{$\begin{array}{l}\text { Corresponding author: } \\
\text { Andre Calitz, } \\
\text { andre.calitz@nmmu.ac.za }\end{array}$} \\
\hline \multicolumn{2}{|c|}{$\begin{array}{l}\text { Dates: } \\
\text { Received: } 14 \text { Dec. } 2016 \\
\text { Accepted: } 23 \text { Mar. } 2017 \\
\text { Published: } 25 \text { July } 2017\end{array}$} \\
\hline \multicolumn{2}{|c|}{$\begin{array}{l}\text { How to cite this article: } \\
\text { Calitz, A.P., Poisat, P., \& Cullen, } \\
\text { M. (2017). The future African } \\
\text { workplace: The use of } \\
\text { collaborative robots in } \\
\text { manufacturing. SA Journal of } \\
\text { Human Resource } \\
\text { Management/SA Tydskrif vir } \\
\text { Menslikehulpbronbestuur, } \\
\text { 15(0), a901. https://doi.org/ } \\
\text { 10.4102/sajhrm.v15i0.901 }\end{array}$} \\
\hline \multicolumn{2}{|c|}{$\begin{array}{l}\text { Copyright: } \\
\text { (C) 2017. The Authors } \\
\text { Licensee: AOSIS. This } \\
\text { is licensed under the } \\
\text { Creative Commons } \\
\text { Attribution License. }\end{array}$} \\
\hline \multicolumn{2}{|l|}{ Read online: } \\
\hline 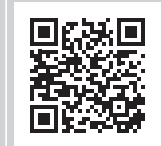 & $\begin{array}{l}\text { Scan this QR } \\
\text { code with your } \\
\text { smart phone or } \\
\text { mobile device } \\
\text { to read online. }\end{array}$ \\
\hline
\end{tabular}

Orientation: Industry 4.0 promotes technological innovations and human-robot collaboration (HRC). Human-robot interaction (HRI) and HRC on the manufacturing assembly line have been implemented in numerous advanced production environments worldwide. Collaborative robots (Cobots) are increasingly being used as collaborators with humans in factory production and assembly environments.

Research purpose: The purpose of the research is to investigate the current use and future implementation of Cobots worldwide and its specific impact on the African workforce.

Motivation for the study: Exploring the gap that exists between the international implementation of Cobots and the potential implementation and impact on the African manufacturing and assembly environment and specifically on the African workforce.

Research design, approach and method: The study features a qualitative research design. An open-ended question survey was conducted amongst leading manufacturing companies in South Africa in order to determine the status and future implementation of Cobot practices. Thematic analysis and content analysis were conducted using AtlasTi.

Main findings: The findings indicate that the African businesses were aware of the international business trends, regarding Cobot implementation, and the possible impact of Cobots on the African work force. Factors specifically highlighted in this study are fear of retrenchment, human-Cobot trust and the African culture.

Practical implications and value-add: This study provides valuable background on the international status of Cobot implementation and the possible impact on the African workforce. The study highlights the importance of building employee trust, providing the relevant training and addressing the fear of retrenchment amongst employees.

\section{Introduction}

\section{Key focus of the study and background}

The term 'Industry 4.0' originated in Germany when the German government launched a hightech strategy initiative, which promotes the computerisation of manufacturing facilities. Industry 4.0 is labelled as the Fourth Industrial Revolution and marks the current trend of automation and data exchange in manufacturing technologies. Industry 4.0 includes cyber-physical systems, the Internet of Things (IoT) and cloud computing. Industry 4.0 creates a 'smart factory' within modular-structured smart factories, where cyber-physical systems monitor physical processes.

In 1920, the word 'robot' was invented by the Czech writer Karel Capek and used in his sciencefiction stage play Rossum's Universal Robots. In 1954, the first industrial robot arm was patented, followed by an era of 'caged' robots during 1960-2000. In 1999, published research indicated that intelligent assist devices, today known as Cobots, could reduce ergonomic concerns and improve safety, quality and productivity (Akella et al., 1999). The first UR5 collaborative robot able to operate safely alongside humans was installed in December 2008, deploying the robot right alongside humans. This launched Universal Robots as a major player in the industrial automation market (UR, 2016). During 2012-2016, collaborative robotics become standard practice with large manufacturing businesses. In 2016, ISO published ISO/TS 15066, which provided guidelines on how to ensure the safety of human workers in collaborative robotic systems (UR, 2016). Today, over 8400 Cobots are installed in over 55 countries, and in South Africa, vehicle manufacturers are increasingly investing in Cobots.

Collaborative robots (Cobots) are designed to work alongside human workers on assembly lines, assisting humans with a variety of assembly and manufacturing tasks. The automotive industry 
has been investigating and increasingly implementing Cobots on assembly lines. Cobots are increasingly being used as collaborators to humans in various contexts and industries, and researchers have investigated actions to promote higher team cohesiveness (Strohkorb \& Scassellati, 2016).

Businesses are introducing Cobots because they can be placed alongside humans in small-spaced assembly lines. Cobots are more affordable and easily trainable and programmable than existing industrial robots, and they are flexible to handle short runs, repetitive and boring jobs and ergonomically challenging tasks. Small- and medium-sized enterprises (SMEs) are eager to adopt this new technology as Cobots have become more affordable. Most professional service robots are collaborative by design, hence Cobots are not just for SMEs or for manufacturing use. Offices, homes, laboratories, warehouses, farms, distribution centres, hospitals and health care facilities are all employing service robots to help them do their jobs better. The market is openended but current uses for Cobots include machine tending, material handling, assembly tasks and packaging. Cobots can also pick, place, count and inspect. As more Cobots are deployed, more uses will be discovered.

Industrial robots were caged to keep humans safe and out of harm's way. Service robots (Cobots) are meant to safely leave the cage whilst doing tasks for humans. Cobots come in all shapes and sizes and have integrated sensors and soft and rounded surfaces for safety purposes to reduce the risk of impact, pinching and crushing. The biggest safety feature of Cobots is their force-limited joints, which are designed to sense forces due to impact and quickly react thereto. Businesses increasingly use industrial robots in their manufacturing operations to reduce labour costs and increase competitiveness (Technavio, 2016).

In a human-machine study conducted by MIT researchers at a BMW factory, the results indicated that teams made of humans and Cobots collaborating efficiently can be more productive than teams made up of either humans or Cobots alone. The study found that the co-operative process reduced human idle time by $85 \%$ (Tobe, 2015). BMW already has 7500 industrial robots installed in their factories and are presently testing Universal Robots (UR3 = \$23 000, UR5 =\$35000 and UR10 = \$45 000) alongside humans specifically in ergonomically challenging assignments. Other automobile manufacturers including Mercedes-Benz, Audi and Toyota have implemented Cobots in production facilities. MercedesBenz indicated that they switched to smaller and safer robots working alongside humans for greater flexibility (Gibbs, 2016). Mercedes-Benz are thus moving away from maximising automation, whilst increasing the role of the human in the industrial process (Payne, 2016).

Small- and medium-sized enterprises are increasingly adopting and investing in this new technology. This segment will see massive growth in the next few years (Tobe, 2015). Internationally, there has been a rise in bot-sourcing. This stems from the current practices of outsourcing labour to countries such as China and India. Businesses internationally are reshoring their manufacturing operations back to home countries due to rising labour costs and declining costs of industrial robots and the availability of Cobots. These developments have created a platform for a new breed of industrial employee to emerge, where workers learn to work side by side with the latest generation of Cobots (Hollinger, 2016).

\section{Research objectives}

The primary purpose of this study is to investigate the current use and future implementation of Cobots in the African manufacturing environment. In line with this purpose, the main objective of the research is to conduct a survey amongst leading manufacturing companies in South Africa and selected companies in Africa in order to determine the current and future planned implementation of Cobot practices. The main research question is: What are the main issues relating to the African workforce regarding the implementation of Cobots? The core research problem relates to a gap in empirical research on the status of Cobot practices in Africa and the possible implications for the African workforce.

\section{The potential value-add of the study}

Internationally, human-robot collaboration (HRC) on the manufacturing assembly line has been implemented in numerous advanced production environments. The value added by this study is assessing the extent to which Cobot practices on the assembly lines in African manufacturing businesses are currently being investigated and implemented. The issues Human Resource (HR) practitioners must take cognisance of when introducing these new business practices are highlighted in this study.

\section{What will follow?}

The next section is a review of the literature, outlining research on the use of Cobots on assembly lines in manufacturing environments and integrating it with this research study. This is followed by a discussion of the theoretical foundation on human-robot collaboration, theory identification, the research design, research findings, discussion and conclusions.

\section{Synthesis and critical evaluation of the literature \\ Industry 4.0}

Industry 4.0 is also known as the Fourth Industrial Revolution and is the current international trend of automation, the IoT and data exchange in manufacturing environments. Introducing these new technologies into a modern manufacturing environment is known as creating 'smart factories'. Industry 4.0 refers to the combination of several major innovations in digital technology transforming the manufacturing sectors. In 2015, PricewaterhouseCoopers (PwC) surveyed more than 2000 companies from 26 countries in the industrial production sectors and found that companies 
are experiencing both cost reductions and revenue gains from their advanced digitisation efforts (Geissbauer, Vedsø \& Schrauf, 2016). Three aspects of digitisation lie at the heart of an Industry 4.0 approach, namely the full digitisation of a company's operations, the redesign of products and services, and closer interaction with customers (Geissbauer, Vedsø \& Schrauf, 2016). Industry 4.0 will affect the following areas: services and business models, reliability and continuous productivity, IT security, machine safety, product lifecycles, the industry value chain and, most importantly, the workers' education and skills (training) and socio-economic factors (Ind4.0, 2016).

\section{Industrial robots}

General Motors introduced the first industrial robots in 1961. Today robots perform a variety of tasks in automotive manufacturing plants, including welding and spray painting (Gordon, 2016). Robots free humans from repetitive tasks and working under difficult circumstances, such as amongst noxious fumes in vehicle paint shops. Industrial robots generally consist of a robotic arm, control cabinet, control panel and other peripherals. They are used for various manufacturing operations, including welding (Figure 1), painting, pick and place, palletising, cutting, assembly, product testing and inspection. During the period 19602000 , industrial robots were generally 'caged', but presently Cobots work in a collaborative working environment alongside humans (UR, 2016).

Market research analysts have predicted the industrial robotics market in the United States to grow rapidly at an impressive compounded annual growth rate (CAGR) of more than $12 \%$ over the forecast period 2016-2020 (Technavio, 2016). They have further predicted the global assembly line robots market to grow steadily at a CAGR of $7 \%$ during the period 2016-2020. The need for flexibility in plant operations is one of the primary drivers of the market.

\section{Cobots}

Collaborative robots are a robotic device intended to interact physically with humans in a shared workspace (Peshkin \&

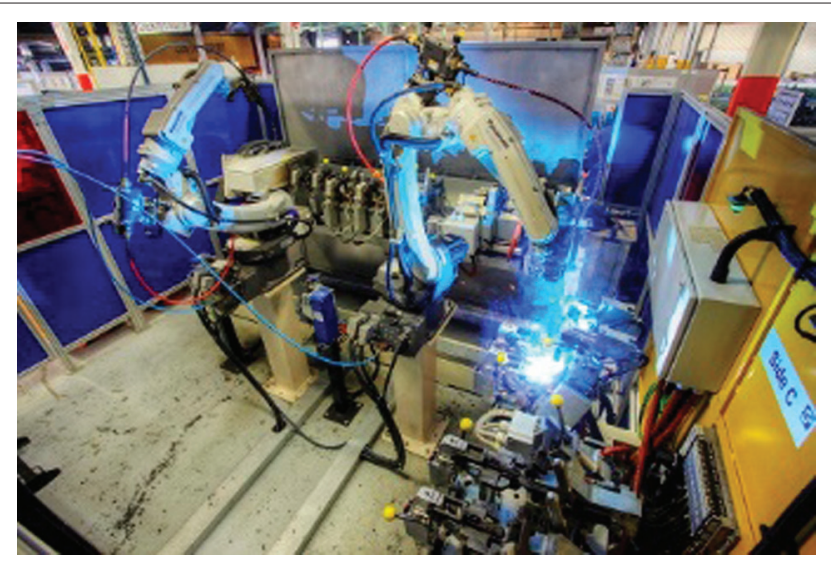

Source: S4. (2016). S4 Integration. Retrieved December 02, 2016 from http://www.S4.co.za FIGURE 1: Industrial welding robots.
Edward, 1999). A Cobot can manipulate objects and perform specified tasks in collaboration with human workers (Colgate, Wannasuphoprasit \& Peshkin, 1996). Cobots are designed to work alongside human workers in production environments, assisting humans with a variety of tasks (Tobe, 2015). The updated characteristics of traditional industrial robots and Cobots are displayed in Table 1, indicating that Cobots are more flexible, easy to programme using graphical user interfaces, less expensive and are safety and easily redeployable (Djuric, Urbanic \& Rickli, 2016).

The European Union SME Robot programme commenced in 2005 with the goal of increasing worker productivity to avoid the off-shoring of labour-intensive tasks (Tobe, 2015). Businesses are introducing Cobots because they can interact with humans in a shared workspace, performing tasks in collaboration with humans. Cobots are becoming increasingly affordable and can easily be trained. They are flexible to handle short runs and complete repetitive and boring jobs and ergonomically challenging tasks (Tobe, 2015). The use of Cobots minimises the margin of error in operations in comparison with manual labour and they do not require any supervision. Cobots further reduce product failure and wastage and their adoption enables companies to minimise direct and overhead costs (Hollinger, 2016).

A leading vendor in the Cobot market, ASEA Brown Boveri (ABB), recently launched YuMi, a two-arm Cobot capable of advanced manual tasks (Figure 2). ABB's YuMi is a desk-top robot that has flexible hands and incorporates a parts feeding system, a camera-based part location and automated control system. YuMi can collaborate side by side or across a workbench with human workers, allowing businesses to obtain optimal performance from humans and Cobots working together (MCISA, 2017). Figures 1 and 2 indicate that Cobots are presently being designed and manufactured in different shapes and sizes. Cobots (Figure 2) have integrated sensors and soft rounded surfaces for safety

TABLE 1: Comparison between traditional industrial robots and Cobots.

\begin{tabular}{|c|c|}
\hline Traditional industrial robots & Cobots \\
\hline Fixed installation & Flexible and portable \\
\hline $\begin{array}{l}\text { Periodic, repeatable tasks; infrequent } \\
\text { changes }\end{array}$ & $\begin{array}{l}\text { Frequent task changes; tasks repeated } \\
\text { infrequently }\end{array}$ \\
\hline On-line and off-line programming & $\begin{array}{l}\text { On-line instruction and supported by } \\
\text { off-line methods }\end{array}$ \\
\hline Traditional programming languages & $\begin{array}{l}\text { Easily programmable using } \\
\text { graphical user interfaces }\end{array}$ \\
\hline Not easy to teach & Easy to teach \\
\hline $\begin{array}{l}\text { Rarely interact with the worker, } \\
\text { only when programmed }\end{array}$ & $\begin{array}{l}\text { Frequent interaction with the worker, } \\
\text { even force or precision assistance }\end{array}$ \\
\hline Workers and robot separated by a fence & Workspace sharing with workers \\
\hline Cannot interact with people safely & Interacts with people safely \\
\hline $\begin{array}{l}\text { Profitable only with medium } \\
\text { to large lot size }\end{array}$ & Profitable even at small lot level \\
\hline Small or big and fast & Small and slow \\
\hline $\begin{array}{l}\text { Cannot reduce cost and footprint } \\
\text { to justify new applications }\end{array}$ & $\begin{array}{l}\text { Reduce cost and footprint to } \\
\text { justify new applications }\end{array}$ \\
\hline No requested risk assessment & Requested risk assessment \\
\hline $\begin{array}{l}\text { Usually } 6 \text { axes with last } \\
\text { three intersecting in wrist }\end{array}$ & $\begin{array}{l}\text { Usually } 6 \text { and } 7 \text { axes with } \\
\text { many offsets }\end{array}$ \\
\hline
\end{tabular}

Source: Adapted from Djuric, A.M., Urbanic, R.J., \& Rickli, J.L. (2016). A framework for collaborative robot (CoBot) integration in advanced manufacturing systems. SAE International Journal of Materials and Manufacturing, 9(2), 457-464 
purposes in order to reduce the risk of impact, pinching and crushing. The biggest safety feature of Cobots is their forcelimited joints, which are designed to sense forces because of impact and quickly respond to the situation (Tobe, 2015).

Cobots are becoming increasingly more affordable and highly adaptable and are easier to train (reprogram) and deploy when compared to industrial robots (Tobe, 2015). Cobots can be programmed using a tablet or simply by moving the arms of the Cobot in the required pattern. Cobots can further easily be moved in a production environment. Fast-Berglund, Palmkvist, Nyqvist, Ekered and Åkerman (2016) compared the Cobots (UR3 and UR5) in final assembly with humans performing the task of fitting O-ring assembly manually. The study found that Cobots have great potential for financial cost savings, human time savings and improved shop floor design. Barclays Capital estimates that the Cobot market could grow by $\$ 3$ billion by 2020 (Hollinger, 2016). Presently, more than 600 UR robots are installed internationally (MCISA, 2017).

The adoption of Cobots has reduced the employee workload drastically. Monotonous jobs such as assembling are now performed by Cobots on the production line, working alongside human co-workers with high productivity and consistent quality. The introduction of Cobots allows workers to focus more on supervising and decision-making roles. The use of Cobots has assisted the automotive industry to a great extent (Technavio, 2016). Cobots are specifically designed for automotive assembly lines. Various models have been designed to assist humans in the final automotive assembly stages of the production of cars (Unhelkar \& Shah, 2015). Smart working environments have been designed to allow true joint actions of humans and Cobots (Lenz, Nair, Rickert \& Knoll, 2008). Cobots now have the capacity to understand the environment they operate in and the actions performed by human co-workers working collaboratively. Coupeté, Moutarde and Manitsaris (2015) have developed a gesture recognition system that allows the Cobot to understand which task is performed by the worker, in order to synchronise their actions.

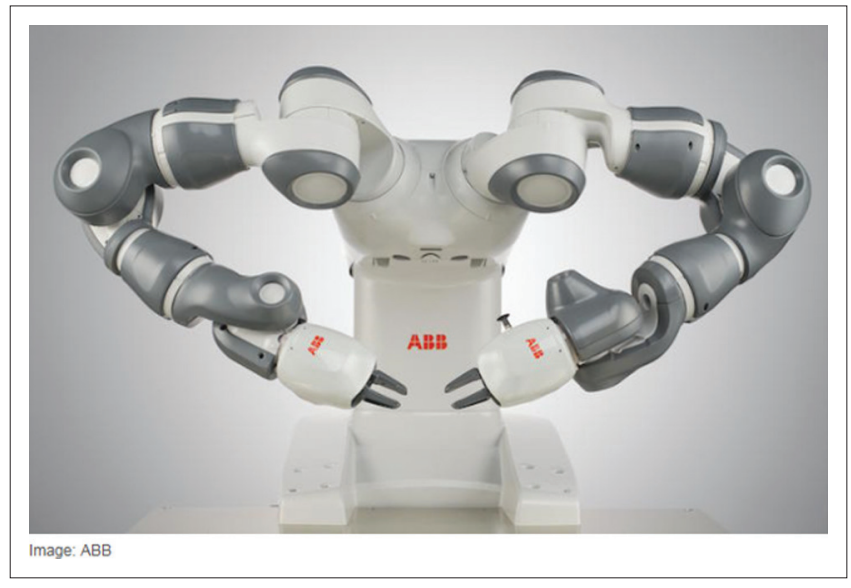

Source: ABB. (2016). Yumi Collaborative robot. Retrieved December 05, 2016, from http:// new.abb.com/products/robotics/industrial-robots/yumi

FIGURE 2: YUMi, a collaborative robot developed by ABB.

\section{Human-robot interaction}

Human-robot interaction (HRI) can be described as humans interacting with Cobots in a production or assembly environment. HRI is a sub-field of human-computer interaction (HCI) (Yanco \& Drury, 2002). Lawton (2016) indicated that collaborative implies working side by side or across a work space with humans whilst performing humanscale tasks (Figure 3). The interaction between humans and robots improves the efficiency and effectiveness of assembly processes, particularly when the robot serves as an intelligent assistant (Kruger, Lien \& Verl, 2009). Modern ergonomic workspaces, where humans and Cobots jointly perform an assembly process in a collaborative environment are being implemented internationally (Faber, Butzler \& Schlick, 2015). Safety and workspace layout are challenges for modern factory automation in promoting human-robot collaboration.

In human-robot interaction, trust is an important factor (Billings et al., 2012). Human-robot trust determines a worker's acceptance of a Cobot in a collaborative work environment. Sanders, Oleson, Billings, Chen and Hancock (2011) explored the theoretical foundations of trust and developed a model of human-robot team trust to provide a deeper understanding of the factors that facilitate the development of human trust in robotic teammates. The three-factor model of trust developed by Billings et al. (2012) indicates that human characteristics, environmental characteristics and robot characteristics influence humanrobot trust. Additional factors include usability, social acceptance, user experience and societal impact (Weiss, Bernhaupt, Lankes \& Tscheligi, 2009). These factors all influence how people experience robots as support for collaborative work and accept them as part of the shop floor and society. Various metrics have been identified that focus on the human, the robot and the system (Murphy \& Schreckenhost, 2013). The human metrics include the metrics of trust, training, mental computation and workload. The robot metrics include time for various operations, self-awareness and how long the robot can go without direct supervision. The system metrics include productivity, efficiency, reliability, safety and coactivity

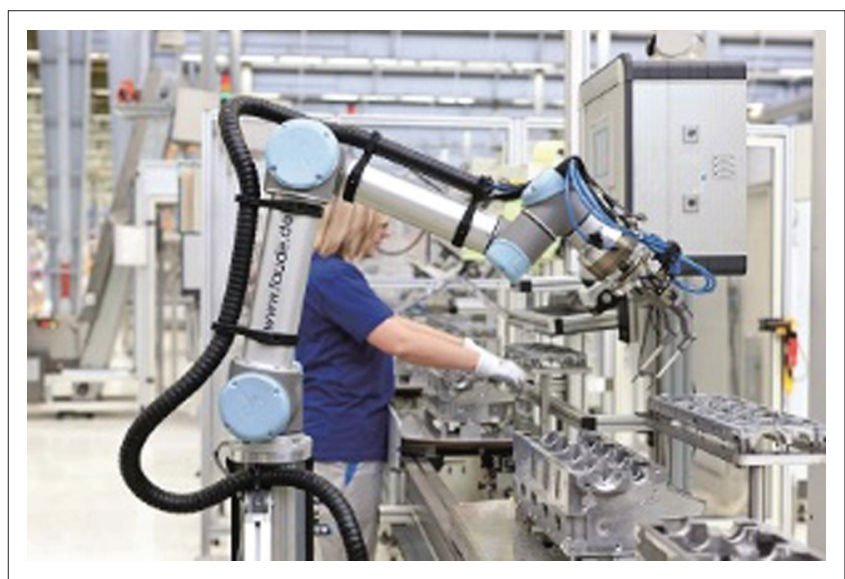

Source: S4. (2016). S4 Integration. Retrieved December 02, 2016 from http://www.S4.co.za FIGURE 3: Human-robot collaboration. 
(Murphy \& Schreckenhost, 2013). Yanco and Drury (2002) have further developed a taxonomy of human-robot interaction, to provide a basis for research in this area. They identified 11 categories that need to be considered when investigating the interaction between humans and robots. Yanco and Drury (2002) highlight the importance of the composition of human-robot teams, decision support for humans and human-robot interaction.

Strategies relating to trust have been researched for manipulating the Cobots in environments where humans and Cobots work together as co-workers. These strategies determine the best outcomes considering both human factors and overall systems performance. Humans completing repetitive tasks on assembly lines and trust of robot models have been proposed, based on human and robot performance (Sadrfaridpour, Burke \& Wang, 2014). The human's trust in the robot determines the degree of autonomy of the robots and hence affects task efficiency and workload. The study (Sadrfaridpour et al., 2014) compared the average trust and workload amongst manual, autonomous and collaborative modes. The collaborative mode showed to have higher average trust with a moderate workload.

\section{Workforce implications}

Industry 4.0 and human-robot collaboration will significantly impact workplace design and commensurately introduce new challenges as a result of the increase in open, virtual work platforms and extensive human-machine interactions (KPMG, 2016). Trust that humans have in the human-robot interaction is a major challenge that should be addressed. Sanders et al. (2011) propose that human (ability or personality), environmental (task or team) and robot characteristics (performance or attribute) are key determinants in promoting human-robot team trust. Their research shows that robot characteristics such as behaviour, type, anthropomorphism and reliability had the largest effect on developing trust. Employees' perceptions of safety in humanrobot interaction are another factor closely related to generating trust (Kagermann, Wahlster \& Helbig, 2013; Sadrfaridpour et al., 2014). Research indicates that trust in robots varies according to culture ( $\mathrm{Li}, \mathrm{Rau} \& \mathrm{Li}, 2010)$ and type of communication (implicit or explicit) within different cultures (Wang, Rau, Evers, Robinson \& Hinds, 2010). This is particularly relevant to South African organisations considering their culturally diverse workforces. Besides the training needs that emanate from human-robot interaction, such as developing trust, safety, ergonomics (human-robot) and employee competencies required to interact or programme the robot; specific focus will be required to approach the aforementioned within a particular cultural framework (South African - an in-depth discussion on culture falls outside the scope of the article).

Employees interacting with robots will be required to be flexible, innovative, perform tasks that are more demanding and may experience tension between the world of artificial intelligence and the world of the workers' own experience
(Kagermann et al., 2013). Workers could experience a loss of control and a sense of alienation from their work as meaningful, reciprocal social interactions decline. Social exchange theory (Emerson, 1976) serves as basis for conceptualising a new psychological-socio-technological relationship between man and artificial intelligence. From the aforementioned, the authors concluded that it is axiomatic that the employee value proposition for employees interacting with robots will have to be considered anew, in particular with regard to the job, working experience, competencies and social system.

\section{Academic theories identified}

Cobotics is a new field of study, which crosses multiple disciplines and examines human-robot collaboration (HRC). $\mathrm{HCI}$ in the field of HRC requires the mention and consideration of academic theories from the disciplines of the social and natural sciences. The knowledge captured in academic theories can provide a framework for practitioners to consider the daily implementation of collaborative work environments and strategic planning (Cornelissen, 2005). Theories help to organise thoughts, generate coherent explanations and achieve understanding (Hambrick, 2007). However, Hambrick argues that some papers simply contribute to our knowledge and/or understanding and possibly have a high likelihood of stimulating future research that will substantially alter managerial theory and practice or the development of a new theory. The authors of this exploratory study note that the field of Cobotics possibly needs the development of a new theory and recommends it as a topic for future research. Whetten's (1989) guidelines in terms of what constitutes a theoretical contribution will be considered. These include the following: What constructs or factors should be considered? How are they related? Why which indicates the underlying dynamics that justify the construct selection and their causal relationships; and who, where and when - where the temporal and contextual constructs set the boundaries of generalisability.

This exploratory research considered many theories, which included the technology acceptance model, theory of behavioural trust and theory of computational trust. Gligor, Jeannette and Wing (2011) in their work on trust in networks of humans and computers propose that a general theory of trust should focus on the establishment of new trust relations where none were possible before. This focus would help create new economic opportunities by increasing the pool of usable services, removing co-operation barriers amongst users and at the very least, taking advantage of network effects. Traditional theoretical frameworks on trust are centred on interpersonal or psychological trust dynamics. A new understanding is that it can also be towards some process or mechanism, in this case robots. Vidyasagar's (1987) research discusses systems theory and robotics in terms of design for ease of control, design for flexible effects and a systematic approach. In the natural sciences, scientific models are taught to give credence to theory and historical examples are used to teach the method (Cockshott, 2011). 
The diffusion of innovation theory was also considered as it describes the process through which new innovations and ideas become diffused and adopted within wider social networks (Murray, 2009). Diffusion of innovation seeks to explain how innovations are taken up in a population. An innovation is an idea, behaviour or object that is perceived as new by its audience. Robinson (2012) offers three insights:

- What qualities make an innovation spread?

- The importance of peer-peer conversations and peer networks.

- Understanding the needs of different user segments.

Innovations tend to be adopted more quickly when they (1) have a relative advantage over existing methods, (2) are compatible with existing values, past experiences and current needs, (3) are simple to understand, (4) can be tried out or played with by potential adopters and (5) are observable, such that adopters can see the results for themselves (Rogers, 2004). Diffusion is the process through which an innovation is communicated. Communication involves people exchanging and creating information that results in collective understanding about the innovation. Key to this theory would be to get 'early adopters' who would enable their peer's acceptance of the Cobots. The theory includes the innovation-decision process, which comprises five stages: (1) knowledge, (2) persuasion, (3) decision, (4) implementation and (5) confirmation (Rogers, 1983). Criticism of this theory is that it is assumed that all new methods are helpful and productive and should therefore be adopted. Some innovations may not fit an organisation's culture and the negative may outweigh the positive (Miles, 2012).

\section{Research design, approach and method \\ Research approach}

The research design was exploratory and explanatory. The research was qualitative in nature, utilising a questionnaire with open-ended questions. AtlasTi was used for the thematic analysis and content analysis of the respondent's responses to individual questions.

\section{Research method}

Participants and sample: South African automotive manufacturing organisations were selected for the study considering that internationally this sector is in the advanced stages of Cobot implementation on assembly lines. IT managers and production managers are generally responsible for technological implementations in the production environment. IT managers at the South African motor vehicle manufacturers were approached and requested to distribute and complete the survey. In addition, production managers from other selected manufacturing environments, such as component manufacturers, were approached and requested to complete the survey. Six African businesses were identified and approached via email. The data collection resulted in 12 usable questionnaires, which represents a $42 \%$ response rate.
The measuring instrument: An e-mail-based survey was used in this study. The first part of the survey related to demographic data collection. The second part provided 10 open-ended questions relating to the use of Cobots internationally and locally and the planned future use of Cobots in the present production environment. Specific questions relating to the African workforce and Cobot implementation were further specified. These questions related to implications for the African workforce, culture, trust and communication and training.

\section{Cobot questionnaire}

The questionnaire was compiled from literature, and questionnaires were used to evaluate HRC. The three-factor model of trust indicates that human characteristics, environmental characteristics and robot characteristics influence human-robot trust (Billings et al., 2012). This research was used as a basis for the compilation of the questionnaire.

Validity: Subject matter experts and the NMMU statistician confirmed the face validity of the questionnaire.

\section{Questionnaire pre-testing}

The proposed research questionnaire underwent pre-testing with academics and three IT managers. A stratified sampling procedure ensured that employees from the identified occupational groups were represented appropriately. A total of 12 fully completed responses were received over a period of 4 weeks.

\section{Research procedure}

A list of automotive manufacturers in South Africa was obtained and the IT manager or Chief Information Officer (CIO) was identified. The respondents were contacted telephonically and requested to complete the survey. A convenience snowball sampling technique was used in this study. The survey was emailed to the respondents, and they were requested to complete the survey and forward it to other possible respondents they know working in the field of study. A Cobot supplier in South Africa assisted with the distribution amongst their client base.

\section{Statistical analysis}

The results were analysed using both thematic analysis and content analysis. The responses were analysed using the software package AtlasTi and the results visually presented as word clouds. Word clouds display the most prominent or frequent words in a body of text. Data visualisation software generally includes word cloud analysis and display and has been utilised in this study.

\section{Results and discussion}

The respondents included IT managers, plant or system engineers, production managers and a technology specialist. The companies represented in this survey are two automobile 
manufacturers, production automation suppliers and two component manufacturers. One respondent represents a business that is a leading worldwide supplier of automation technologies and another respondent worked for a business selling Cobots in South Africa who regularly conduct customer demonstrations. Five respondents had experience regarding the use of Cobots in manufacturing and seven had no experience but knowledge and an understanding of the field. Seven of the businesses represented in the survey have international parent or holding companies, already utilising Cobots in manufacturing.

The thematic analysis of responses indicated that knowledge was gained through watching product demo videos, reading articles and actual experience implementing robotic solutions (Figure 4). Five manufacturing plants in South Africa have implemented Cobots, whilst six businesses are investigating the implementation into their businesses. South African businesses are experimenting with 'proof of concept phase implementations' of Cobots and two businesses have installed Cobots on the assembly and manufacturing lines. Respondents further indicated that they are 'identifying suitable pilot application environments' (Participant 4, Male, IT Manager), 'numerous applications are being investigated' (Participant 6, Male, Plant Manager) and Industry 4.0 'will become standard for all plants and manufacturing facilities worldwide' (Participant 11, Male, Technology Specialist).

Eight of the respondents indicated that they think the use of Cobots is a viable option for South African and African companies. One respondent indicated that 'South Africa is a developing country and any technology advancements could only be advantageous to drive economic growth' (Participant 3, Female, Business Developer). Respondents further indicated that the introduction of Cobots could improve work precision, allow workers to work smarter, and enhance human performance and that Cobots generally do not replace humans, but assist them in mundane and routine tasks (Figure 5).

The reasons four respondents indicated that the use of Cobots in manufacturing is not a viable option for the South African and African companies were:

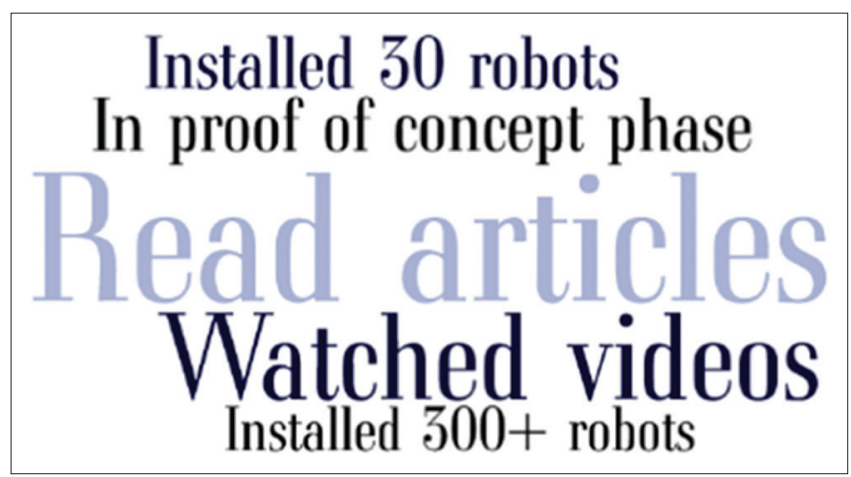

FIGURE 4: Sources of Cobot information and implementation.
- the cost of the technology being perceived as high

- labour costs are relatively cheap in comparison with international countries

- a generally technically inexperienced workforce

- a poorly educated and highly unionised workforce.

The major challenges for South African and African businesses that introduce Cobots at the organisation level are resistance from unions, technology implementation costs, change management, training and addressing the employees' increased fear of unemployment (Figure 6). One respondent indicated that 'low volume producers will accept Cobots as they are more cost competitive than semi-skilled labour in S.A' (Participant 6, Male, Plant Manager). Time will, however, force business into alternative sources of labour - such as Cobots. The respondent further indicated that 'the major impact is on unemployment in the medium to long term, as Cobots/robots become more common in manufacturing' (Participant 6, Male, Plant Manager).

The major challenges for South African and African businesses that introduce Cobots at the employee level are fear of redundancy, retrenchment and increased unemployment (Figure 7). The challenges can be addressed by education and training. The introduction of a collaborative working model is suggested in order to address the lack of skills workers have working alongside robots. A respondent indicated that 'the impact on employee motivation could be the single biggest challenge. Additionally retrenchment due to implementation of technology is a challenge to achieve' (Participant 6, Male, Plant Manager).

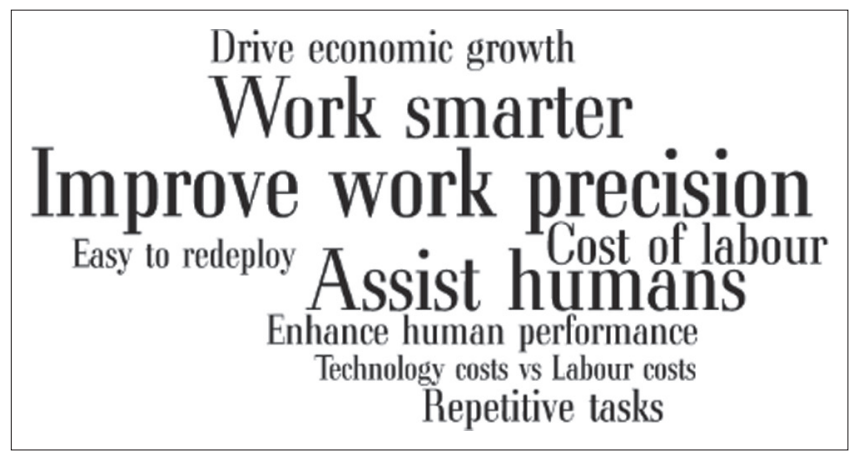

FIGURE 5: Benefits of introducing Cobots.

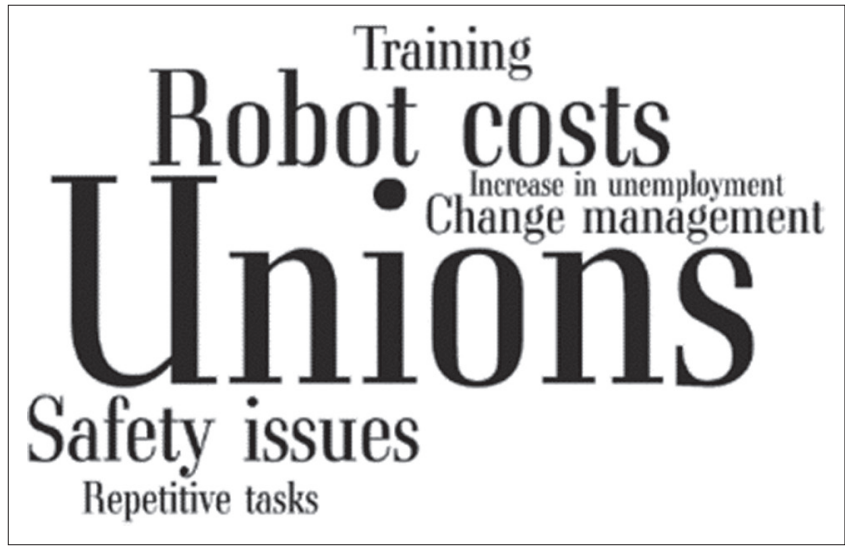

FIGURE 6: Challenges at organisational level. 
Training and education were identified as important factors for the introduction of Cobots (Ind4.0, 2016). The major training needs for the introduction of Cobots at senior management level are business benefits $(n=3)$, technology awareness and benefits (Figure 8). A respondent indicated that 'knowing what is available in the market place, how it can provide a competitive advantage and be beneficial to the company' (Participant 3, Female, Business Developer) are the major issues for senior management.

The major training needs for the introduction of Cobots at employee level are greater technical training, trust and safety, understanding robotic functionality and employee loneliness (Figure 9). Respondents further indicated that the 'understanding of how the robots function and their own role in the production line' (Participant 3, Female, Business Developer) is important. 'In the long term there might be some emotional support needed as the job might become lonely without other humans in the proximity' (Participant 9, Male, Operations Manager) and 'basic computer skills and good hand-eye coordination' (Participant 11, Male, Technology Specialist) are major training needs for employees.

\section{Unemployment Rear of redundancy Keeping up with technology Training Education Employee motivation}

FIGURE 7: Challenges at employee level.

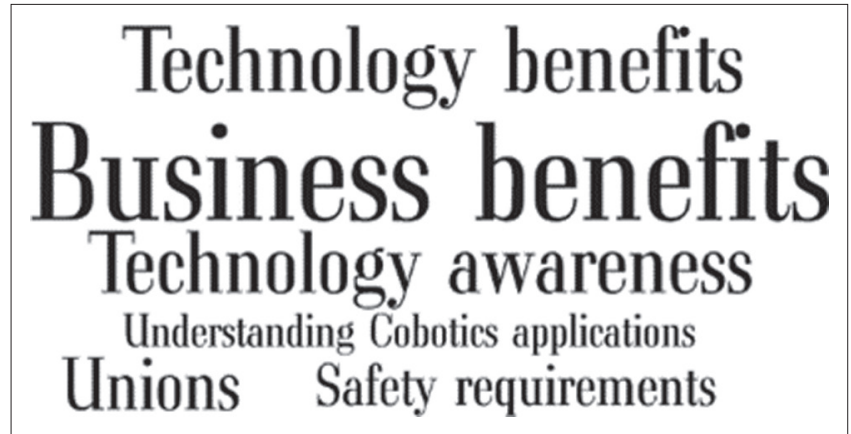

FIGURE 8: Senior management training needs.

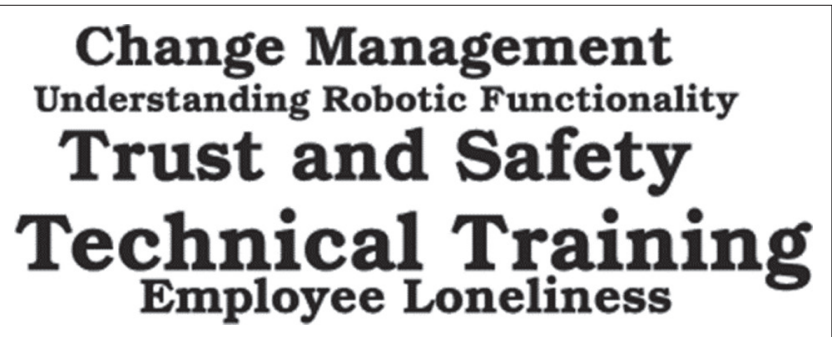

FIGURE 9: Employee training needs.
Respondents indicated that the following human characteristics (Figure 10) will have the most significant impact on promoting human-robot trust: ability $(n=4)$, trust $(n=3)$, new technology acceptance $(n=2)$ and competency $(n=2)$. Trust, specifically in terms of safety, is highlighted by the response that 'they need a high-level understanding of how the Cobot functions to be able to trust it' (Participant 11, Male, Technology Specialist).

Respondents generally indicated that communication $(n=3)$ is an important environmental characteristic that will have a significant impact on promoting human-robot trust for collaboration (Figure 11). Other characteristics are culture and continuous learning, 'so that new technologies can be embraced to their fullest potential' (Participant 11, Male, Technology Specialist).

Respondents indicated that the following robot characteristics will have the most significant impact on promoting humanrobot trust: perform as designed $(n=3)$, function to standard specification, dependability and seeing the benefit of working collaboratively (Figure 12). Respondents emphasised that 'a robot must perform $100 \%$ as expected/designed' (Participant 1, Male, IT Manager), 'humans need confidence that the robot will function to the standard specification for

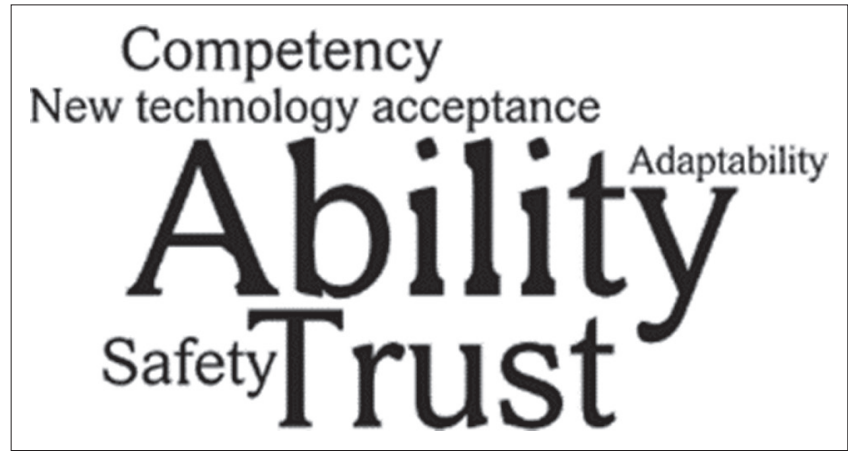

FIGURE 10: Human characteristics.

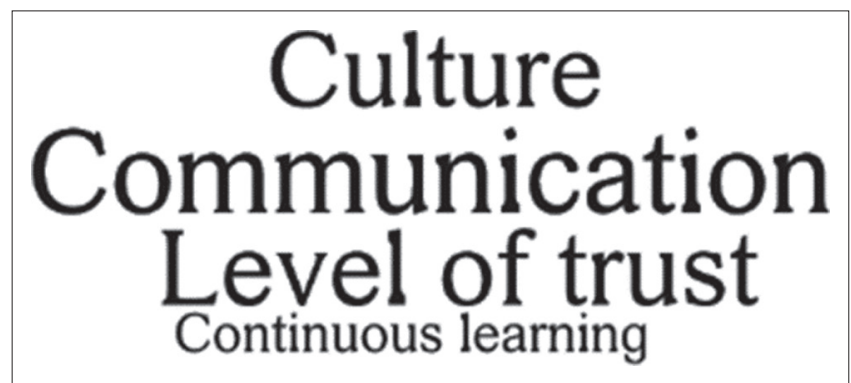

FIGURE 11: Environmental characteristics.

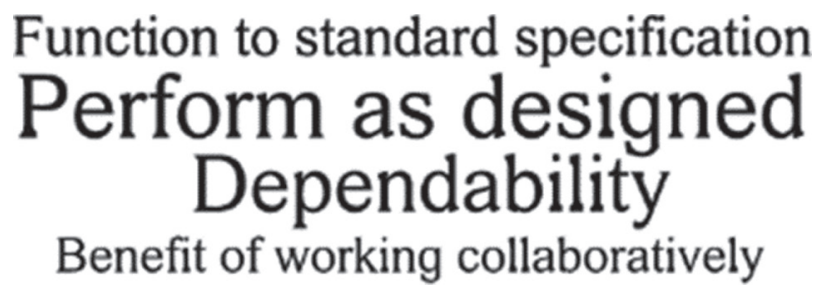

FIGURE 12: Robot characteristics. 
which it was designed' (Participant 3, Female, Business Developer) and 'when the employee realises how dependable the robot is', robot trust will increase. Dependability was also mentioned, 'if the worker feels the robot depends on his input, rather than him on the robot, the worker will feel more value and in control' (Participant 11, Male, Technology Specialist). Other respondents stated that:

Personality - I have read of some studies that indicate humans react better to robots mimicking certain human behaviours. I do however believe that this would not be easily implemented in the workplace as it is normally all about cycle time and volume of production. (Participant 9, Male, Operations Manager)

The psychological demands and roles placed on employees interacting with robots are mainly job security $(n=3)$ and stress $(n=2)$. Respondents highlighted the 'fear of being replaced' and the 'fear of the unknown' (Figure 13). Regarding stress, respondents highlighted 'The pressure to perform in a completely structured manner without reasoning ability' (Participant 1, Male, IT Manager) and 'Stress in the sense the robot could replace the person' (Participant 6, Male, Plant Manager).

The physical demands and roles placed on employees (Figure 14) interacting with robots are reduced physical demands $(n=2)$ and reduced demanding tasks $(n=2)$. Respondents indicated that with increased competitiveness, 'the person will want to compete with the robot, or act completely incorrectly, resulting in forms of sabotage' (Participant 6, Male, Plant Manager). 'The person might find it difficult to keep up with the robot' $^{\prime}$ (Participant 11, Male, Technology Specialist) and 'the application of Cobots could increase the employee's health since it can lead them to handle tasks in a proper physical position' (Participant 12, Male, Robot Researcher).

The intellectual demands and roles placed on employees interacting with robots are mainly intellectual improvements,

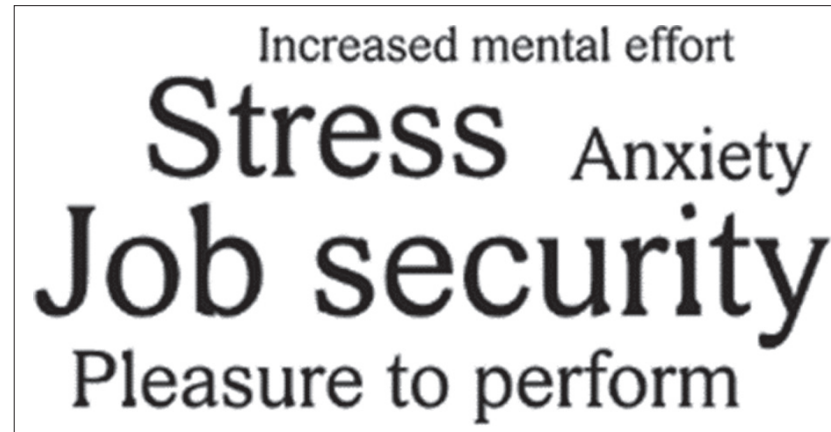

Fear of the unknown

FIGURE 13: Psychological demands.

\section{Reduced demanding tasks Reduced physical demands Increased competativeness}

FIGURE 14: Physical demands. performing more challenging tasks and learning new behaviours (Figure 15). Intellectual improvement due new technological advancements and exposure to new technologies were emphasised. Respondents highlighted that employees must 'understand process flows and the required outputs of robots' (Participant 3, Male, Business Developer). Employees will work and operate at a 'more mental level and at a less physical level' (Participant 7, Male, Customer Support Manager).

The ideal employee traits or characteristics for interacting with Cobots and flourishing in the envisaged work environment are willingness to learn $(n=4)$, supporting training and upskilling, accepting change, being technologically inclined and adapting to new technologies (Figure 16). The two respondents who indicated that humans working with Cobots are introverted have both implemented Cobots in their businesses and have gained experience with workers working in these environments. Further employee traits include an independent and self-assured individual.

The majority of the respondents $(n=9)$ indicated that they believe culture will have an impact on the human-robot interaction (Figure 17). The major themes regarding culture that were identified were education and literacy, resistance to new and unknown technological innovations and work ethic. The threat to unemployment was mentioned by two respondents.

Interestingly, the automotive manufacturer respondents, some of whom have introduced Cobots, did not think 'culture' had a major impact on HRI. One respondent indicated that 'culture impacts the relationship between humans and should be taken into consideration for interaction with robots as well' (Participant 3, Female, Business Developer). A respondent indicated that 'African people do not resist adapting to and using new technologies, just see their

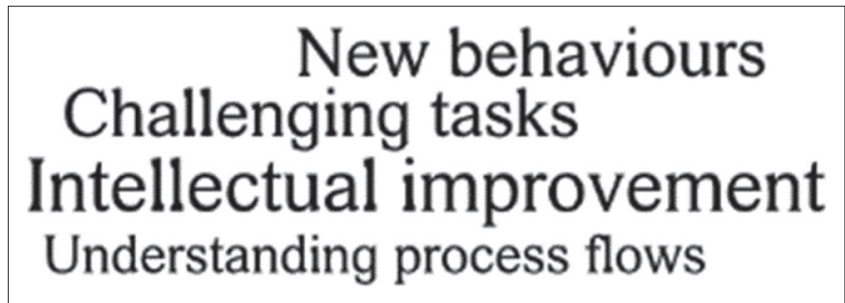

FIGURE 15: Intellectual demands.

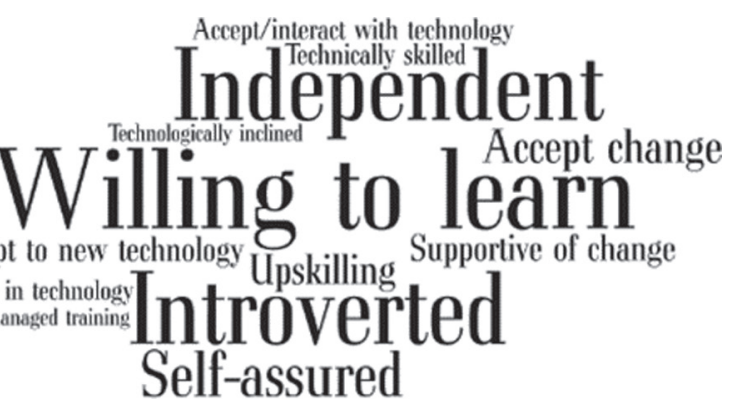

FIGURE 16: Employee traits. 


\section{Resistance to change Threat to employment Work ethic Exposure to technology \\ Education and Literacy \\ Collectivist culture}

FIGURE 17: Cultural impact.

dependence on mobile applications' (Participant 12, Male, Robot Researcher). 'It is my belief that they would find it hard to accept a robot rather than working with a colleague (African culture very focused on relationships)' (Participant 11, Male, Operations Manager).

\section{Managerial implications}

Humans and Cobots have essential collaborative abilities and advantages regarding industrial assembly processes. Cobots can conduct repetitive and monotonous assembly steps, and humans can adapt to new situations and anticipate problems. Management must consider modern ergonomic workspaces (Faber, Butzler \& Schlick, 2015) where humans and Cobots jointly perform an assembly process in a collaborative environment. Managers further need to be aware of the declining costs of industrial robots and the increased availability of Cobots. Bot-sourcing will become an important continuous improvement activity for businesses in the manufacturing industry.

Findings of the research suggest that South African and African businesses do not have the option of not following Industry 4.0, international trends and collaborative work practices. Automotive suppliers and component manufacturers have implemented collaborative work spaces and the extensive use of Cobots. One automotive manufacturer has installed over 300 Cobots in the past months and provided valuable input into the management of human resources in a collaborative work environment.

Culture plays a significant role in developing trust in robots. The type of communication required for adoption varies between cultures. Therefore, managers of the African workforce must take cognisance of the fact that the manufacturing industry internationally implementing Cobots might investigate automation differently, in the human-Cobot collaborative work environment. Cobots are increasingly viewed as teammates; however, an African workforce that must still establish trust, having a culture of human-human collaboration and fear of unemployment, will require new human resource management practices. Acceptance of a Cobot by its human team members will ultimately determine the success of human-Cobot collaboration in a modern work environment.

\section{Limitations of the study and directions for future research}

This research was conducted in Africa; however, only responses from South African companies were obtained. No responses were received from the six respondents identified and emailed in Africa. A more representative sample from Africa must be obtained. The study is an exploratory study and further research needs to be conducted on the African workforce to address the issues highlighted in this study, namely trust, collaboration, job security and technical training. A suitable theory for human-Cobot collaboration, encompassing the major aspects of this diverse field, needs to be investigated. In addition, the best business practices and lessons learned by the automotive manufacturers who have successfully implemented collaborative work environments in Africa need to be explored.

\section{Conclusion}

Collaborative robots are intended to physically interact with humans in a shared workspace, assisting humans with a variety of tasks. Cobots are becoming more affordable and highly adaptable and can easily be redeployed in different work environments. They are designed alongside humans in a shared workspace. Robots have made little impact outside manufacturing, and it will be a gradual process before robots replace human jobs in the service, transportation or construction sectors (Gordon, 2016). However, research conducted by Technovio (2016) indicates that it has been observed that robots and specifically Cobots have become necessary in every industry that uses production processes and systems.

One of the goals of Universal Robots (UR, 2016) is to make robot technology accessible to SMEs (MCISA, 2017). SMEs, including auto component manufacturers, are generally reluctant to make heavy investments in robots; however, reduced Cobotic costs and the challenges faced by managing manual labour, including unions, will change the adoption of Cobots in the next 5 years. The SME market will become a major driver in the installation of Cobots because they represent over 6 million businesses worldwide and nearly $70 \%$ of the world's manufacturing sector (Tobe, 2015).

The introduction of Cobots into assembly and manufacturing lines will expand automation across the plant floor, which will increase worker safety and efficiency (Technavio, 2016). As more Cobots are deployed, more uses will be discovered. The market acceptance of Cobots, including drones, is expected to be a significant driver in non-industrial robotic growth. The Cobot sector is expected to increase roughly tenfold between 2015 and 2020, reaching over \$1 billion from approximately $\$ 95$ million in 2014. The global Cobot market is expected to grow at a CAGR of $51 \%$ until 2019 (Technavio, 2016; Tobe, 2015). Barclays Capital forecasts that the Cobot market will grow to 150 million units in 2020, representing a $\$ 3.1$ billion global market (Lawton, 2016). 
African manufacturing industries must consider the new category of human-friendly Cobots that will work safely with humans. African managers must align their workforce strategy with their business strategy. The mobility and flexibility that Cobot solutions provide will create a sustainable work environment of the future.

\section{Acknowledgements Competing interests}

The authors declare that they have no financial or personal relationships that may have inappropriately influenced them in writing this article.

\section{Authors' contributions}

A.P.C. and P.P. were principal researchers who conducted the empirical research. A.P.C. analysed the responses using AtlasTi. M.C. provided relevant theories and language editing. All authors contributed to writing the final article.

\section{References}

ABB. (2016). Yumi Collaborative robot. Retrieved December 05, 2016, from http:// new.abb.com/products/robotics/industrial-robots/yum

Akella, P., Peshkin, M., Colgate, E., Wannasuphoprasit, W., Nagesh, N., Wells, J., et al. (1999). Cobots for the automobile assembly line. In Proceedings of the 1999 IEEE International Conference on Robotics \& Automation (ICRA99), 10-15 May 2009 International Conference on Robotics \& Autor
(pp. 728-733), Detroit, MI. New York: IEEE.

Billings, D.R., Chen, J.Y.C., \& Hancock, P.A. (2012). Human-robot interaction: Developing trust in Robots. In HRI'12 Human-robot interaction (pp. 109-110). New York: ACM.

Cockshott, P. (2011). Competing theories: Wrong or not even wrong? Vlaams Marxistisch Tijdschrift, 45(2), 97-103.

Colgate, J.E., Wannasuphoprasit, W., \& Peshkin, M.A. (1996). СОВOTS: Robots for collaboration with human operators. In Proceedings of the International Mechanical Engineering Congress and Exhibition, 17-22 November 2011 (Vol. 58, pp. 433-439). Atlanta, Georgia: DSC.

Cornelissen, J.P. (2005). Toward an understanding of the use of academic theories in public relations practice. Public Relations Review, 26(3), 315-326. https://doi. org/10.1016/S0363-8111(00)00050-3

Coupeté, E., Moutarde, F., \& Manitsaris, S. (2015). A user-adaptive gesture recognition system applied to human-robot collaboration in factories. In MOCO'16 (p. 239) July 05-06, 2016. Thessaloniki, GA, Greece.

Djuric, A.M., Urbanic, R.J., \& Rickli, J.L. (2016). A framework for collaborative robot (CoBot) integration in advanced manufacturing systems. SAE International Journal of Materials and Manufacturing, 9(2), 457-464.

Emerson, R.M. (1976) Social exchange theory. Annual Review of Sociology, 2, 335-362.

Faber, M., Butzler, J., \& Schlick, C.M. (2015). Human-robot cooperation in future production systems: Analysis of requirements for designing an ergonomic work system. Procedia Manufacturing, 3, 510-517. https://doi.org/10.1016/j.promfg. 2015.07.215

Fast-Berglund, Å., Palmkvist, F., Nyqvist, P., Ekered, S., \& Åkerman, M. (2016) Evaluating Cobots for final assembly. In 6th CIRP Conference on Assembly Technologies and Systems (CATS), 16-18 May 2016 (Vol. 44, pp. 175-180). Technologies and Systen
Gothenburg, Sweden.

Geissbauer, R., Vedsø, J., \& Schrauf, S. (2016). A strategist's guide to Industry 4.0. Retrieved November 25, 2016, from http://www.strategy-business.com/article/AStrategists-Guide-to-Industry-4.0?gko=7c4cf

Gibbs, S. (2016). Mercedes-Benz swaps robots for people on its assembly lines. The Guardian. Retrieved October 9, 2016, from https://www.theguardian.com/ technology/2016/feb/26/mercedes-benz-robots-people-assembly-lines

Gligor, V., \& Jeannette, M., Wing, J.M. (2011). Towards a theory of trust in networks of humans and computers. Presented at the 19th International Workshop on Security Protocols, 28-30 March 2011 (pp. 28-30). Cambridge, UK.

Gordon, R. (2016). Why robots will not decimate human jobs. Retrieved November 24, 2016, from http://buildakingdombusiness.com/?p=732

Hambrick, D.C. 2007. The field of management's devotion to theory: Too much of a good thing? Academy of Management Journal, 50(6), 1346-1352. https://doi. org/10.5465/AMJ.2007.28166119
Hollinger, P. (2016, May 4). Meet the Cobots: Humans and robots together on the factory floor. The Financial Times.

IND4.0. (2016). Industry 4.0. Retrieved November 20, 2016, from https://en.wikipedia. org/wiki/Industry_4.0

Kagermann, H., Wahlster, W., \& Helbig, J. (2013). Recommendations for implementing the strategic initiatives INDUSTRIE 4.0. Final Report of the Indistrie 4.0 Working Group. Frankfurt: National Academy of Science and Engineering.

Kruger, J., Lien, T.K., \& Verl, A. (2009). Cooperation of human and machines in assembly lines. CIRP Annals - Manufacturing Technology, 58, 628-646.

KPMG. (2016). Global CEO outlook. Retrieved November 16, 2017, from https://hbr. org/2016/10/globalization-robots-and-the-future-of-work

Lawton, J. (2016). The collaborative robot market is set for phenomenal growth (part 1). Rethinking Robots, September 28, 2016.

Lenz, C., Nair, S., Rickert, M., \& Knoll, A. (2008). Joint-action for humans and industria robots for assembly tasks. In Proceedings of the 17th IEEE International Symposium on Robot and Human Interactive Communication (pp. 130-135), Technische Universität München, Munich, Germany. August 1-3, 2008.

Li, D., Rau, P., \& Li, Y. (2010). A cross-cultural study: Effect of robot appearance and task. International Journal of Social Robots, 2, 175-186. https://doi.org/10.1007/ s12369-010-0056-9

Miles, J.A. (2012). Management and organization theory. A Jossey-Bass Reader. San Francisco, CA: John Wiley \& Sons, Inc.

MCISA. (2017). The latest robots are friendly. Motion Control in South Africa. Retrieved March 15, 2017, from http://www.motioncontrol.co.za/55852n

Murphy, R.R., \& Schreckenghost, D. (2013). Survey of metrics for human-robot interaction. In 8th ACM/IEEE International Conference on Human-Robot Interaction (HRI), 3-6 March 2013. New York: IEEE.

Murray, C.E. (2009). Diffusion of innovation theory: A bridge for the research-practice gap in counseling. Journal of Counseling and Development, The American Counseling Association. 87, 108-116.

Payne, J. (2016). Mercedes-Benz swaps robots for people on its assembly lines. Retrieved November 26, 2016, from http://robohub.org/mercedes-benz-swapsrobots-for-people-on-its-assembly-lines-the-guardian/

Peshkin, M., \& Edward, J.C. (1999). CoBots (invited). Industrial Robot, 26(5), 335-341. https://doi.org/10.1108/01439919910283722

Robinson, L. (2012). A summary of diffusion of innovations. In Changeology: How to enable groups, communities and societies to do things they've never done before (pp. 1-272). Cambridge, UK: Green Books.

Rogers, E.M. (1983). Diffusion of innovations (3rd ed.). New York: Free Press.

Rogers, E.M. (2004). A prospective and retrospective look at the diffusion model. Journal of Health Communication, 9, 13-19. https://doi.org/10.1080/10810730490271449

Sadrfaridpour, B., Burke, J., \& Wang, Y. (2014). Human and robot collaborative assembly manufacturing: trust dynamics and control. In Proceedings of the Workshop on Human-Robot Collaboration Industrial Manufacturing. RSS, 12-16 July 2014 (pp. 1-6). Berkeley, CA: UC

Sanders, T., Oleson, K.E., Billings, D.R., Chen, J.Y.C., \& Hancock, P.A. (2011). A model of human-robot trust. Theoretical model development. Proceedings of the Human Factors and Ergonomics Society Annual Meeting, 55(1), 1432-1436.

Strohkorb, S., \& Scassellati, B. (2016). Promoting collaboration with social robots. In HRI '16: The Eleventh ACM/IEEE International Conference on Human Robot Interaction, 08-10 March 2016 (pp. 639-640). New York: IEEE.

S4. (2016). S4 Integration. Retrieved December 02, 2016 from http://www.S4.co.za

Technavio. (2016). Sample- Industrial Robotics Market in the USA. Retrieved November 20, 2016, from http://www.technavio.com

Tobe, F. (2015). Why co-bots will be a huge innovation and growth driver for robotics industry. Retrieved October 10, 2016, from, http://spectrum.ieee.org/automaton/ robotics/industrial-robots/collaborative-robots-innovation-growth-driver

Unhelkar, V.V., \& Shah, J.A. (2015). Challenges in developing a collaborative robotic assistant for automotive assembly lines. In HRI'15 Extended Abstracts (pp. 239-240), March 2-5, 2015, Portland, OR, USA.

UR. (2016). Universal robots and ... the Cobots. Retrieved October 26, 2016, from, http://www.universal-robots.com/about-universal-robots/news-centre/historyof-the-cobots/

Vidyasagar, M. (1987). System theory and robotics. IEEE Control Systems Magazine, 7(3) (1987), 16-17. https://doi.org/10.1109/MCS.1987.1105283

Wang, L., Rau, P.L., Evers, V., Robinson, B.K., \& Hinds, P. (2010). Whenin Rome: The role of culture and context in adherence to robot recommendations. In Proceedings of the 5th ACM/IEEE International Conference on Human Robot Interaction, 02-05 March 2010 (pp. 359-366). New York: IEEE.

Weiss, A., Bernhaupt, R., Lankes, M., \& Tscheligi, M. (2009). The USUS evaluation framework for human-robot interaction. In AISB2009: Proceedings of the Symposium on New Frontiers in Human-Robot Interaction, 06-09 April 2009. UK: SSAISB: Edinburgh.

Whetten, D.A. (1989). What constitutes a theoretical contribution? Academy of Management Review, 14, 490-495.

Yanco, H.A., \& Drury, J.L. (2002). A taxonomy for human-robot interaction. In AAAl Fall Symposium on Human-Robot Interaction, 15-17 November 2002 (pp. 111-119). Technical Report FS-02-03. CA: AAAI. North Falmouth, Massachusetts. 